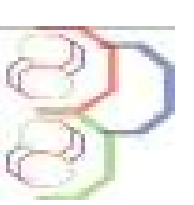

\title{
Évaluation de deux variétés de riz (CK73 et CK90) à la Toxicité Ferreuse et à quelques contraintes biotiques à Yamoussoukro (Côte d'Ivoire)
}

\author{
A.J. Gnago', K.T. Kouadio² ${ }^{\star}$, V.E. Tia ${ }^{3}$, A.P. Kodro ${ }^{1}$, A.V. Goulivas ${ }^{4}$ \\ ${ }^{1}$ Institut National Polytechnique Félix Houphouët-Boigny, Département de Formation et de Recherche Agriculture et \\ Ressources Animales, Laboratoire de Zoologie Agricole et d'Entomologie, B.P. 1313 Yamoussoukro, Côte d'Ivoire \\ ${ }^{2}$ Institut National Polytechnique Félix Houphouët-Boigny, Département de Formation et de Recherche Agriculture et \\ Ressources Animales, Laboratoire de Phytopathologie et de Biologie Végétale, B.P. 1313 Yamoussoukro, Côte \\ d'Ivoire \\ 3Université Péléforo Gon Coulibaly, Unité de Formation et de Recherche des Sciences Biologiques, B.P. 1328 \\ Korhogo, Côte d'Ivoire \\ ${ }^{4}$ Ministère de l'Agriculture, PROPACOM, BP 951 Bouaké, Côte d'Ivoire \\ * : E-mail auteur correspondant : tkouadiothed@gmail.com
}

Original submitted in on $31^{\text {st }}$ March 2017. Published online at www.m.elewa.org on $30^{\text {th }}$ April 2017 https://dx.doi.org/10.4314/jab.v112i1.8

\section{RESUME}

Objectif : La toxicité ferreuse est un désordre nutritionnel associé à de fortes concentrations de fer pouvant entraîner des pertes de rendement sur le riz. L'objectif de cette étude est d'évaluer le comportement de deux variétés de riz CK 73 et CK 90 à la toxicité ferreuse ainsi qu'à d'autres contraintes biotiques en riziculture irriguée sur le périmètre de Petit Bouaké à Yamoussoukro en Côte d'Ivoire.

Méthodologie et résultats : Un bloc de Fisher complètement randomisé avec 3 répétitions a été mis en place où les variétés à vulgariser (CK 73 et CK 90) ont été comparées à la variété WITA9 comme témoin sensible. Les notes de sévérités relatives à la toxicité ferreuse, à la pyriculariose, et à l'helminthosporiose ont été mesurées. Les scores moyens des variétés testées à la toxicité ferreuse étaient de 3,$5 ; 3,9 ; 4,2$ respectivement pour les variétés CK90, WITA9 et CK73. Les rendements moyens en paddy des variétés CK73, CK 90 et WITA9 étaient respectivement de 2,7 T/ha, 2,2 T/ha et 4,5 T/ha. La tolérance des variétés testées à la Pyriculariose foliaire a été bonne au cours de cette étude avec des scores moyens de 1,$6 ; 1$ et 1 respectivement pour CK 73, CK90 et WITA9. Quant à l'Helminthosporiose, les notes de sévérité étaient de 5,4;3,2 et 2,6 respectivement pour les variétés CK 73, CK90 et WITA9.

Conclusion et applications : L'évaluation des deux (02) variétés de riz à diffuser en Côte d'Ivoire (CK73 et CK90) a permis de confirmer leur tolérance à la toxicité ferreuse sur le site de Petit Bouaké à Yamoussoukro. En outre, la variété WITA 9 déjà diffusée et bien appréciée par les producteurs, a aussi présenté une tolérance moyenne. Les rendements en paddy des variétés CK73 et CK 90 sont acceptables au regard des rendements moyens fournis par les fiches techniques de références (3-4T/ha).

Mots clés : riz, variété, toxicité ferreuse, Helminthosporiose, Pyriculariose, Côte d'Ivoire 
Evaluation of two rice varieties (CK73 and CK90) on iron toxicity and some biotic stress in Yamoussoukro (Ivory Coast)

\section{ABSTRACT}

Objective: The iron toxicity is a nutrient disorder associated with high iron concentrations may be lead to a significant impact on rice yield. The objective of this study is to evaluate the behaviors of two rice varieties CK73 and CK90 on iron toxicity and some biotic stress in irrigated lowlands on the perimeter of Petit Bouaké at Yamoussoukro in Ivory Coast.

Methodology and results: A randomized complete block design with three replications was implemented in order to compare CK73 and CK90 introduced varieties with WITA9 as control which was already known. Iron toxicity, blast disease and leaf blight scores were recorded. The mean of iron toxicity visual score of tested varieties are 3.5, 3.9, 4.2 for CK90, WITA9 and CK73 respectively. Average yield of paddy for tested varieties are 2.7 T/ha, 2.2 T/ha, 4.5 T/ha for CK73, CK90 and WITA 9 respectively. The tolerance of varieties to blast disease was good with mean scores of 1.6; $1 ; 1$ for CK73, CK90 and WITA9 respectively. Furthermore, the mean of leaf blight visual score of varieties are $5.4 ; 3.2 ; 2.6$ for CK 73, CK90 and WITA9 respectively.

Conclusion and applications: The evaluation of two rice varieties (CK73 and CK90) in order to disseminate in Ivory Coast was able to confirm their tolerance on iron toxicity in the site of Yamoussoukro. Furthermore, WITA9 which was already disseminated showing a moderate level of tolerance. Paddy yields of the two new varieties are acceptable against the specification sheets (3-4 T/ha).

Keywords: rice, varieties, iron toxicity, blast disease, leaf blight, Ivory Coast

\section{INTRODUCTION}

Le riz (Oryza sativa L.) est la deuxième céréale, après le maïs, la plus cultivée et consommée dans le monde (FAOstat, 2014). En Côte d'Ivoire, cette denrée est la plus consommée avec une moyenne annuelle par habitant de plus de $85 \mathrm{~kg}$ (ONDR, 2016). La production locale est de $1400.000 \mathrm{~T}$ pour un besoin total de $1700000 \mathrm{~T}$ par an (ONDR, 2016). Cette production est dominée par le riz pluvial qui occupe des surfaces cultivées, contrairement au riz irrigué qui occupe que $5 \%$ des superficies (ONDR, 2016). Malgré les efforts consentis par les pouvoirs publics et la recherche pour mettre au point des variétés de riz performantes et des itinéraires techniques adaptés aux différents types de rizicultures, les rendements demeurent faibles. Les causes de la faible productivité du riz cultivé localement en Côte d'Ivoire sont multiples. Parmi elles, les maladies comme la panachure jaune du riz due au Rice yellow mottle virus (RYMV), la pyriculariose (due à Magnaporthe grisea) et l'helminthosporiose (due à Bipolaris oryzae) constituent des contraintes majeures (Amoncho et al., 2009 ; Bouet, 2008 ; Bouet et al., 2015). En outre, la riziculture en condition de bas-fond (avec ou sans contrôle de l'eau) est souvent confrontée au problème de toxicité ferreuse (Cherif et al., 2009). C'est un désordre nutritionnel associé à de fortes concentrations de fer dans la solution du sol (Fageria et al., 2008). Les conditions réductrices que l'on rencontre couramment dans les sols engorgés de bas-fond sont précurseurs de la toxicité ferreuse par la solubilisation dans la solution du sol de la quasitotalité du fer sous forme ferreux (Fe2+) (Cherif et al., 2009). Au delà d'une concentration de l'ordre de $300 \mathrm{ppm}$, il provoque des dysfonctionnements sur le riz (yellowing, bronzing), malgré la capacité de cette plante à créer une réoxydation partielle autour des racines, par des transferts d'oxygène en provenance des parties aériennes (Becker et Asch, 2005 ; Audebert, 2006a). Ce fer ferreux $\left(\mathrm{Fe}^{2+}+\right.$ ) est alors absorbé en abondance par les racines et se concentre dans les feuilles. II ralentit la croissance, réduit le tallage, par conséquent affecte considérablement le rendement. Les pertes de rendement peuvent en moyenne aller jusqu'à $54 \%$ (Cherif et al., 2009). Pour y remédier, certains auteurs ont proposé diverses pratiques agricoles notamment le blocage du mouvement du fer 
provenant des plateaux en drainant les champs pour les débarrasser des excédents de fer, l'apport de fertilisants et l'utilisation de variétés de riz tolérantes aux fortes concentrations de fer dans le sol (Audebert, 2006b ; Ethan et al., 2011). Toutefois, l'utilisation de variétés tolérantes est sans doute la méthode la plus appropriée à la situation socioéconomique de la plupart des producteurs. Ainsi plusieurs travaux de sélection et de diffusion de variétés tolérantes à la toxicité ferreuse sont menés

\section{MATERIEL ET METHODES}

Site d'étude : L'étude a été réalisée à Yamoussoukro (latitude $6^{\circ} 49$ ' $\mathrm{N}$; longitude $5^{\circ} 16^{\circ} \mathrm{O}$ ), au centre de la Côte d'Ivoire à $240 \mathrm{Km}$ au nord de la ville d'Abidjan. Cette zone est dite de transition forêt/savane. Les sols sont de type ferralitique et humifère au niveau des plateaux alors que sur les plaines et les bas-fonds, ils sont rougeâtres, jaune rougeâtres et sablo-argileux avec une présence d'éléments gravillonnaires (ANADER, 2004). Le climat est de type tropical de transition avec une pluviométrie de type bimodal caractérisée par quatre (04) saisons dont une grande saison de pluie de mars à juillet et une petite saison d'août à octobre. Ces deux saisons de pluie sont intercalées par deux saisons sèches. Les précipitations moyennes annuelles sont de 1022,2 mm d'eau pour 71 jours de pluie par an. La température est d'environ 27,5 ${ }^{\circ} \mathrm{C}$ avec une humidité relative variant entre de $75 \%$ à $85 \%$ mais pouvant chuter pour atteindre $40 \%$ en période d'harmattan (décembre à février) (ANADER, 2004). L'étude a été menée sur le périmètre rizicole de Petit Bouaké (Yamoussoukro) d'octobre 2015 à février 2016. par des centres de recherches en Afrique de l'Ouest (Sikirou et al., 2015). C'est dans ce cadre que les variétés CK 73 et CK 90 ont été testées sur le périmètre de Petit Bouaké, à Yamoussoukro considéré comme une zone à toxicité ferreuse (WARDA, 2006), afin d'évaluer leur comportement à la Toxicité Ferreuse. En outre, quelques contraintes biotiques telles que l'helminthosporiose, la pyriculariose et la panachure jaune du riz ont également été évaluées.

Cette mise en place de l'essai a coïncidé avec la saison sèche incluant l'harmattan.

Matériel végétal : Le matériel végétal est constitué de deux variétés de riz, CK 73 et CK 90, originaires de la Guinée et fournies par le Programme de Productivité Agricole en Afrique de l'Ouest (PPAAO/WAAPP) exécuté par le Fonds Interprofessionnel pour la Recherche et le Conseil Agricole (FIRCA). A cela, s'ajoute la variété améliorée WITA 9 développée par le Centre du Riz pour l'Afrique (AfricaRice) et très appréciée en Côte d'Ivoire. Les semences de cette variété ont été fournies par I'ONDR.

\section{Méthodes}

Dispositif expérimental: L'expérimentation a été réalisée selon un dispositif en blocs complets randomisés à trois répétitions sur une parcelle supposée connue pour sa richesse en fer selon les paysans. Les blocs ( $30 \mathrm{~m} \mathrm{x}$ $20 \mathrm{~m}$ ) ont une superficie de $600 \mathrm{~m}^{2}$ chacun et séparés par des diguettes de $1 \mathrm{~m}$. Les parcelles élémentaires ont une superficie de $200 \mathrm{~m}^{2}(20 \mathrm{~m} \times 10 \mathrm{~m})$ et distantes de 0,50 $\mathrm{m}$.

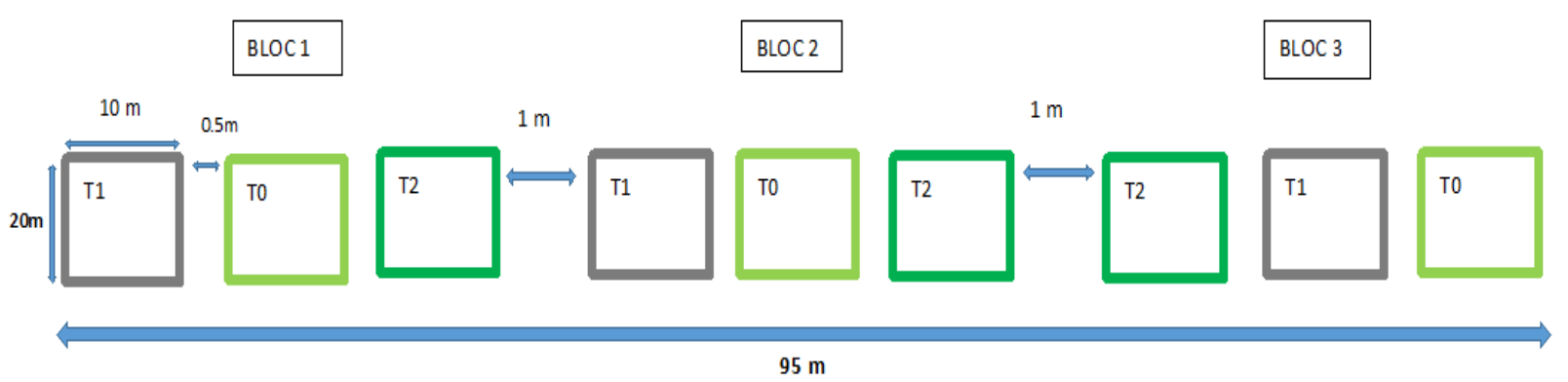

Figure 1 : Schéma du dispositif expérimental à Petit Bouaké à Yamoussoukro, Côte d'lvoire d'octobre 2015 à février 2016. T0 : témoin, variété WITA 9; T1 : variété CK 73 ; T2 : variété CK 90. 

à quelques contraintes biotiques à Yamoussoukro (Côte d'Ivoire)

Prélèvement de sol et dosage du fer avant la mise en

place de l'essai : Dix (10) prélèvements de sol ont été effectués de manière aléatoire par bloc et soigneusement homogénéisés pour constituer un échantillon afin de minimiser les effets dus à l'hétérogénéité de la toxicité. Au total 3 échantillons de sol issus des 3 blocs (B1; B2 ; B3) ont été constitués puis dosés pour la détermination du Fer total. Les analyses ont été effectuées au laboratoire de Pédologie de l'Institut National Polytechnique Félix Houphouët-Boigny de Yamoussoukro, Côte d'Ivoire.

Mise en place des pépinières et repiquage de riz : Les trois variétés de riz ont été mises en pépinière sur le même périmètre à la même date. Les plantules de riz ont été repiquées 12 jours après semis en ligne dans les parcelles suivant des espacements de $0,20 \mathrm{~m}$ entre les lignes et sur les lignes en raison d'un plant par poquet. Le NPK 18-22-22 (100kg/ha) a été utilisé comme engrais de fond après 2 jours de repiquage et l'urée a été épandue au stade tallage à 30 jours après repiquage et au stade montaison à 60 jours après repiquage $(50 \mathrm{Kg} / \mathrm{ha})$. Le Rigold 432 EC (Triclopyr et Propanil) a été appliqué après la levée des plantules comme herbicide sélectifà 7 jours après repiquage.

Mesures réalisées et paramètres calculés: Les mesures ont porté sur la hauteur du riz et le nombre de

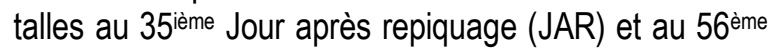
JAR. L'évaluation de la toxicité ferreuse (TF) a été basée sur la note de sévérité ou de score comme proposé par I'IRRI (IRRI, 2013). L'échelle de notation va de 1 à 9 en lien avec le pourcentage de surface foliaire présentant les symptômes typiques de toxicité ferreuse. A partir de ces notes, la variété ayant un score moyen situé dans l'intervalle 1 à 3 est considéré comme résistante, 4 à 6 comme tolérante et 7 à 9 comme sensible (Cherif et al., 2009). Tous les scores de Toxicité Ferreuse ont été enregistrés à 56 JAR. En effet, les symptômes de toxicité ferreuse sont plus visibles entre 40 JAR et 60 JAR (Chérif

\section{RESULTATS}

Évolution du tallage et de la hauteur des plants par variété de riz : La production de talles par variété a été différente (Figure 2a). Cependant, pour toutes les variétés, elle a augmenté jusqu'à 49 JAR suivi d'une dégénérescence de certaines talles. Ainsi à la récolte, le nombre moyen de talles par touffes (pied) de riz a été de 7,$3 ; 8,3$ et 12,4 respectivement pour le CK73, CK90 et WITA 9. L'analyse statistique a révélé un effet hautement significatif du génotype testé $(P=0,0002<0,001)$. Le groupe 1 est constitué de la variété WITA 9 plus et al., 2009). D'autres contraintes ont été notées à savoir l'helminthosporiose, la pyriculariose foliaire et la panachure jaune du riz toujours à partir des échelles de notation de l'IRRI à 56 JAR (IRRI, 2013). A la maturité technologique, le nombre total de talles, de talles fertiles et le nombre de panicules par $\mathrm{m}^{2}$ ont été comptés. En outre, le niveau de fécondité a été donné par le rapport entre le nombre de talles fertiles et le nombre total de talles. Après la récolte, le séchage et le vannage, le poids de 1000 grains et le rendement en paddy ( $\mathrm{T} / \mathrm{ha}$ ) ont été déterminés par des pesées.

Toutes les collectes de données ont été réalisées dans deux (02) carrés de rendement de $1 \mathrm{mX} 1 \mathrm{~m}$ choisis de façon aléatoire dans les différentes parcelles élémentaires. Pour éliminer les effets de bordure, les carrés de rendement sont disposés de sorte à laisser plus d'un mètre par rapport au bord de la parcelle élémentaire. Ces mesures ont porté sur dix (10) touffes de riz par carré dont six (06) prises sur les deux (02) diagonales du carré et quatre (04) prises sur les médianes. Les mesures à la maturité technologique pour le rendement et les composantes du rendement ont été collectées dans un seul carré de rendement.

Analyses statistiques: Toutes les données collectées ont été soumises à des traitements statistiques qui ont permis de les représenter, analyser et interpréter avec le logiciel Microsoft Excel 2007 pour les représentations graphiques et un logiciel libre $R$ version 3.2.3 pour les analyses de variances (ANOVA) au seuil de significativité de $5 \%$ et pour des tests de corrélation. Pour la réalisation des tests ANOVA, les hypothèses d'application à savoir l'indépendance des variables (dans notre cas par la mise en place du dispositif), la normalité et l'homoscédasticité ont été vérifiées. Le test de la différence vraiment significative (HSD) de Tukey a été utilisé pour la ségrégation des moyennes en cas de différence significative au seuil de $5 \%$.

productive que dans le second groupe composé de CK 73 et CK90. Quant à la hauteur moyenne, il a été observé des variations allant de $81,5 \mathrm{~cm}$ (WITA9) à 141,6 $\mathrm{cm}$ (CK90) à 56 JAR (Figure 2b). L'analyse de variance a révélé une différence hautement significative au seuil de $5 \%(P=0,0001<0,001)$ entre les variétés de riz testées. Les variétés de grande taille sont constituées de CK73 $(121 \mathrm{~cm})$ et CK90 et celle de petite taille est WITA9.

Réaction des variétés de riz testées face à la Toxicité

Ferreuse : L'analyse de variance effectuée sur les notes 
de sévérité ou score de la toxicité ferreuse n'a pas montré de différence significative $(P=0,47)$ entre les variétés testées. Cependant, les scores moyens étaient de 3,5 pour la variété CK90 alors que WITA9 et CK73 présentaient respectivement 3,9 et 4,2 . Les trois variétés sont tolérantes ou moyennement résistantes à la toxicité ferreuse vu leur score moyen compris entre 3-5. Les analyses des échantillons de sol pour le dosage du Fer ont révélé des quantités de 731 ppm, 677 ppm et de 491 ppm respectivement pour le bloc 1 , bloc 2 et bloc 3 du dispositif expérimental. Une étude de corrélation entre les scores de toxicité ferreuse et le Fer total par bloc révèle un coefficient de Pearson proche de 1 pour chaque variété. Cependant, ces corrélations ne sont pas significatives pour les trois variétés testées.
Réaction des variétés de riz testées face à I'Helminthosporiose, à la Pyriculariose foliaire et à la Panachure jaune : Concernant l'helminthosporiose, les variétés de riz testées CK73, CK90 et WITA9 ont toutes subi des attaques du champignon avec des notes de sévérités respectives de 5,$4 ; 3,2$ et 2,6 . L'analyse de variance n'a cependant pas révélé de différence significative entre les variétés au seuil de $5 \%(P=$ $0,0648)$. Toutefois, les variétés WITA 9 et CK90 ont montré un bon niveau de résistance. Pour la pyriculariose foliaire, il a été observé très peu d'infection due au champignon. En effet, les notes de sévérités ont été respectivement de 1,$6 ; 1 ; 1$ pour le CK73, CK90 et WITA9. Les variétés ont donc présenté un bon niveau de résistance. Les symptômes typiques de panachure jaune du riz ont été très peu observés sur les variétés testées.

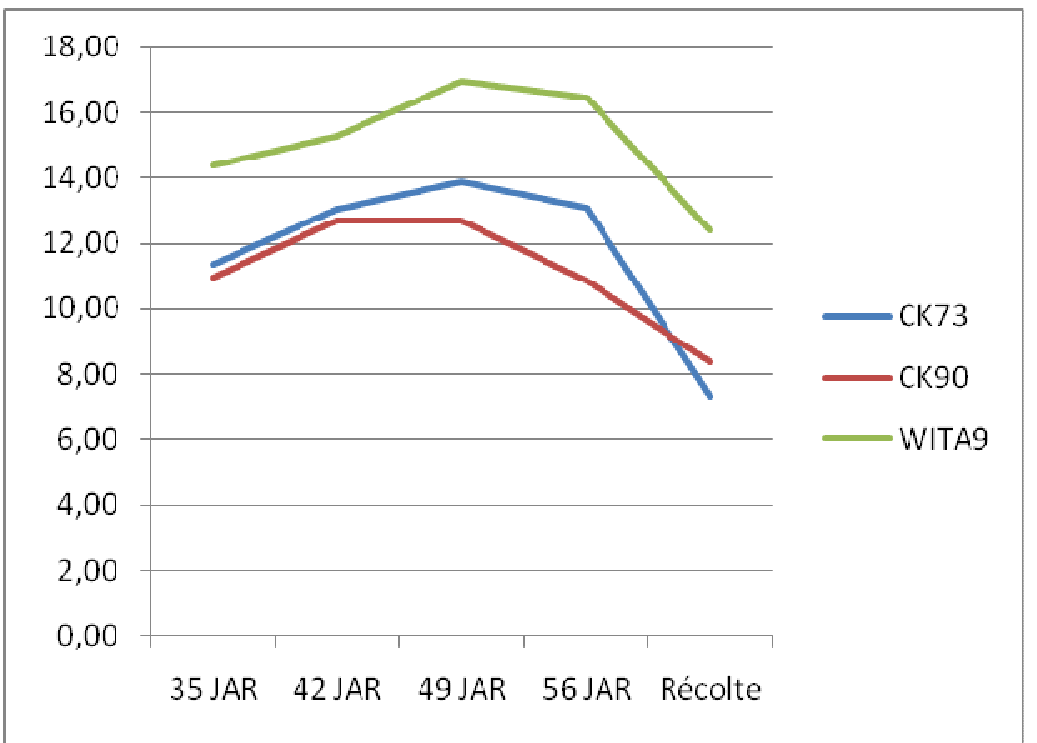

Figure 2a : Evolution du nombre moyen de talle par variétés de riz testées JAR : Jour après repiquage 


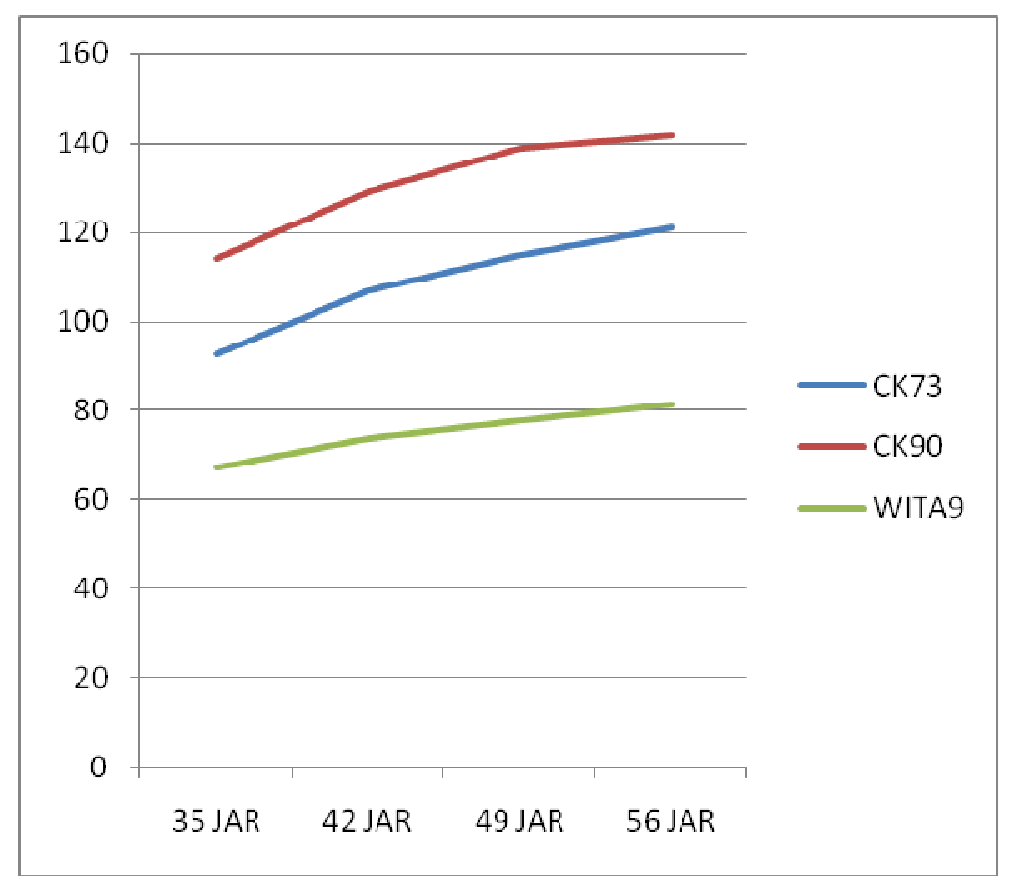

Figure $\mathbf{2 b}$ : Evolution de la hauteur $(\mathrm{cm})$ des plants par variétés de riz testées JAR : Jour après Repiquage

Composantes de rendement et rendement en paddy Niveau de fécondité des talles de riz : Plus le rapport est proche de 1 plus le niveau de fécondité est élevé. Les résultats obtenus ont montré que la variété WITA 9 a présenté le niveau de fécondité le plus élevé $(0,93)$ bien que dans l'ensemble le niveau a été supérieur à 0,70 . La figure 3 illustre les différents niveaux obtenus par chaque variété.

\section{Niveau de fécondité}

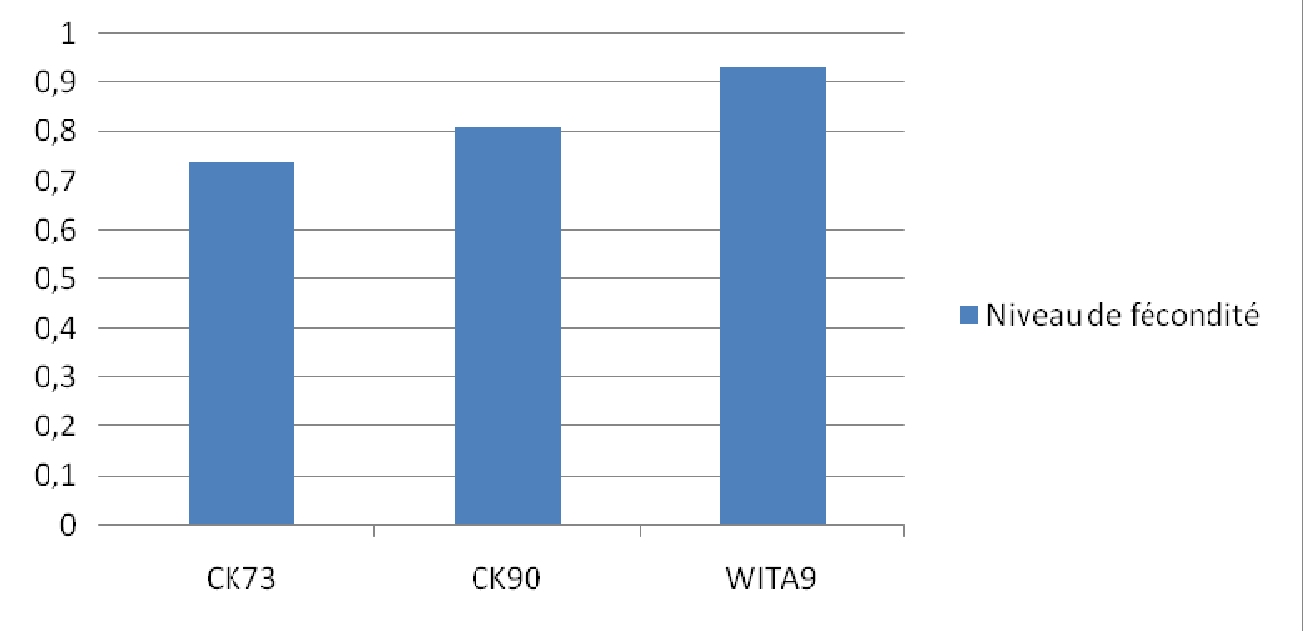

Figure 3 : Niveau de fécondité des variétés CK 73, CK90 et WITA9 testées à Petit Bouaké d'octobre 2015 à février 2016 
Nombre de panicules/m2: L'analyse statistique du nombre moyen de panicules $/ \mathrm{m}^{2}$ a révélé une différence hautement significative entre les variétés au seuil de $5 \%$ $(P=0,0088<0,001)$. Deux groupes de variétés ont été constituées d'une part de WITA $9\left(281,6\right.$ panicules $\left./ \mathrm{m}^{2}\right)$ et d'autre part des variétés CK73 (151,3 panicules $\left./ \mathrm{m}^{2}\right)$ et CK90 (165,3 panicules $\left./ \mathrm{m}^{2}\right)$.

Poids de 1000 grains (g) : Pour le poids de 1000 grains, les variétés testées ont présenté des valeurs allant de 20,1 g (WITA9) à 23,4 g (CK73). Aucune différence significative $(P=0,32)$ n'a été trouvé entre elles.

Rendement du paddy (T/ha) : L'analyse statistique a révélé une différence très hautement significative entre les traitements $(P=0,00016)$. Ainsi selon les résultats deux groupes de variétés ont été formés. La variété WITA9 a été plus productive avec un rendement de 4,5T/ha. Quant au second groupe il est constitué des variétés CK73 et CK90 avec respectivement des rendements de 2,7 et 2,2 T/ha.

Synthèse des résultats: L'ensemble des résultats obtenus à partir des paramètres mesurés sur les trois variétés est résumé au tableau 1. Les variétés ont été classées en groupes homogènes selon le test HSD de Tukey.

Tableau 1 : Synthèse des résultats des paramètres mesurés sur les trois variétés de riz testées sur le site de Petit Bouaké (Yamoussoukro)

\begin{tabular}{|c|c|c|c|}
\hline \multirow[t]{2}{*}{ Paramètres } & \multicolumn{3}{|c|}{ Variétés de riz testées } \\
\hline & CK73 & CK90 & WITA9 \\
\hline Nombre de talles/touffe & $7,3 \pm 0,61 a$ & $8,3 \pm 0,17 a$ & $12,4 \pm 1,0 b$ \\
\hline Hauteur du riz $(\mathrm{cm})$ & $121 \pm 4,91 a$ & $141,6 \pm 10,8 a$ & $81,5 \pm 4,09 \mathrm{~b}$ \\
\hline Sévérité Helminthosporiose & $5,4 \pm 1,73 a$ & $3,2 \pm 0,68 \mathrm{a}$ & $2,6 \pm 0,95 a$ \\
\hline Sévérité Pyriculariose & $1,63 \pm 0,56 a$ & $1 \pm 0 \mathrm{a}$ & $1 \pm 0 \mathrm{a}$ \\
\hline Score Toxicité Ferreuse & $4,2 \pm 0,12 a$ & $3,5 \pm 1,1 \mathrm{a}$ & $3,9 \pm 0,18 a$ \\
\hline Panicules $/ \mathrm{m}^{2}$ & $151,3 \pm 20 a$ & $165,3 \pm 21,5 a$ & $281,6 \pm 56 b$ \\
\hline Masse de 1000 grains (g) & $23,4 \pm 1,9 a$ & $23,3 \pm 4 a$ & $20,1 \pm 0,5 a$ \\
\hline Rendement (T/ha) & $2,7 \pm 0,9 a$ & $2,2 \pm 0,46 a$ & $4,5 \pm 0,18 b$ \\
\hline
\end{tabular}

Les valeurs ayant les mêmes lettres sur la ligne constituent des groupes homogènes

\section{DISCUSSION}

L'évaluation des deux variétés de riz (CK73 et CK90) à diffuser en Côte d'Ivoire a permis de confirmer leur tolérance à la toxicité ferreuse sur le site de Petit Bouaké à Yamoussoukro. En effet, les variétés CK originellement de la Corée du Nord et connues tolérantes à la Toxicité ferreuse font l'objet de plusieurs travaux d'adaptation en station de recherches et en milieu paysan en Afrique de l'Ouest avant leur diffusion (Ouedraogo et Ouadraogo, 2003 ; WARDA, 2006 ; Abdoul, 2006). En outre, la variété déjà diffusée et bien appréciée par les producteurs WITA9, a aussi présenté une bonne tolérance. Cependant, il a été observé une variation de la sévérité de la toxicité ferreuse entre ces variétés testées. La variation de la sévérité des variétés vis-à-vis de la toxicité ferreuse est certainement due à l'existence d'une différence génotypique entre ces dernières. En effet, plusieurs travaux ont montré que les variétés de riz présentent différents comportements vis-à-vis de la toxicité ferreuse (Chérif et al., 2009 ; Onaga et al., 2013 ; Sikirou et al., 2015). Elles peuvent être résistantes, moyennement résistantes ou tolérantes et sensibles.
Mais cette réaction des variétés vis-à-vis de cette toxicité est également sous l'influence des conditions environnementales d'autant plus que le phénotype est l'interaction entre le génotype et l'environnement $\left(P=G^{*} E\right)$. Et cette interaction justifie les travaux d'adaptation et d'adoption des variétés de riz d'une zone agro-écologique à une autre car une variété résistante dans une zone donnée peut être sensible dans une autre. Les résultats des analyses de Fer total dans les blocs d'expérimentation ont révélé que les trois blocs présentaient une quantité importante de fer mais non corrélée significativement aux scores de toxicité ferreuse. $\mathrm{Ce}$ résultat pourrait s'expliquer par le fait que la solubilisation du Fer total en $\mathrm{Fe} 2+$ est fonction de plusieurs paramètres notamment le $\mathrm{pH}$, le potentiel Redox, la capacité d'échange cationique (CEC) et la quantité de potassium échangeable (Audebert, 2006b ; Hodomihou et al., 2011). D'autre part, au cours de l'expérimentation menée dans cette étude, de l'engrais de fond (NPK) et l'engrais de couverture (urée) ont certainement contribué à réduire la solubilisation du Fer 

à quelques contraintes biotiques à Yamoussoukro (Côte d'Ivoire)

total. En effet, des travaux ont montré que l'apport de fertilisants permettait d'atténuer l'effet de la toxicité ferreuse dans les bas-fonds (Audebert, 2006b ; Ethan et al., 2011 ; Hodomihou et al., 2011). Le critère utilisé pour l'attribution de score pour apprécier le comportement des variétés vis-à-vis de la toxicité ferreuse a déjà été rapporté par plusieurs auteurs (Asch et al., 2005 ; Cherif et al., 2007). II est vrai qu'il est subjectif mais la notation des scores comme toute sévérité sur les plants par plus d'une personne permet de réduire les sources d'erreurs relatives à cette appréciation visuelle. Outre la toxicité ferreuse, les variétés de riz ont également été testées visà-vis de quelques maladies parasitaires ou infectieuses dues à des agents pathogènes. C'est ainsi que l'évaluation de la réaction des variétés testées face à l'helminthosporiose a été notée. En effet, cette affection fongique du riz en Côte d'Ivoire jusque-là sans impact économique important prend de l'ampleur (Bouet et al., 2015). Dans cette étude menée, il ressort que les variétés CK 90 et WITA 9 ont montré un bon niveau de résistance à cette maladie alors que la variété CK 73 est moyennement résistante. De même Bouet et al. (2015) avaient relevé qu'en riziculture ivoirienne irriguée à Tiassalé la variété $C K 90$ était résistante et la variété $C K$ 73 relativement sensible. La pyriculariose foliaire est connue comme une contrainte biotique majeure dans la riziculture aquatique (riziculture irriguée et de bas-fonds) en Afrique de l'Ouest (Bouet, 2008 ; Séré et al., 2013; Odjo et al., 2014). Cependant sur le site de Petit Bouaké à Yamoussoukro, soit il n'y a pas eu d'épidémie importante soit les variétés testées ont présenté un bon profil de résistance. Ce résultat pourrait s'expliquer par les conditions climatiques, notamment la saison sèche, pendant la période de l'essai, moins favorable à l'explosion de la maladie. Mais aussi, il peut être en relation avec la faible virulence des isolats de Pyricularia oryzae à Yamoussoukro. Bouet (2008) a déjà mentionné la présence de cinq lignées clonales reparties dans 15 pathotypes en Côte d'lvoire. De même, l'absence d'épidémie due à la panachure jaune de riz ne permet pas de se prononcer sur le comportement des variétés testées. La panachure jaune est plus fréquemment observée en riziculture de bas-fond et constitue une

\section{REMERCIEMENTS}

Les auteurs remercient le Fonds Interprofessionnel pour la Recherche et le Conseil Agricole (FIRCA) à travers le PPAAO/WAAPP et I'ONG INNOV' DEV en Côte d'Ivoire maladie à fort impact économique sur la productivité du riz (Amancho et al., 2008). De ce fait, cette maladie est appelée localement "SIDA du riz». La variété déjà diffusée (WITA 9) en milieu paysan et bien appréciée par ces derniers est connue tolérante à cette maladie. Cependant, la résistance ou la tolérance d'une variété à un agent pathogène donné n'est pas statique et mérite un suivi dans le temps et dans diverses zones agroécologiques. En effet, des prospections menées dans des rizières en Côte d'Ivoire ont montré que la panachure jaune du riz a été observée de façon sporadique sur le WITA 9 excepté une seule localité où une incidence de $5 \%$ a été enregistrée dans une parcelle (Amancho et al., 2008). Sur le plan agronomique, des informations utiles ont été acquises sur les variétés testées. Elles ont présenté un bon développement végétatif illustré par un bon niveau de fécondité des talles. En effet, ce niveau était compris entre 0,73 et 0,93 synonyme d'un nombre important de talles fertiles en rapport avec le nombre total de talles produites. Cependant, la période de réalisation de l'essai ayant coïncidé avec la saison sèche et une décrue du barrage d'alimentation en eau pourraient expliquer les rendements relativement faibles obtenus. En effet, pendant l'essai il a été observé un assèchement des casiers à partir de 60 JAR jusqu'à la récolte. Malgré ce fait, les rendements en paddy des variétés testées sont acceptables au regard des rendements moyens fournis par les fiches techniques de références (3-4 T/ha) et des résultats des travaux de Bouet et al. (2015). En outre, le rendement est un paramètre qui est en partie sous l'influence des conditions culturales (fertilisation, sol, climat, etc.). Alors, en tenant compte que les périodes sèches sont favorables à l'augmentation de la séverité de la toxicité ferreuse et à leur impact sur la productivité (WARDA, 2002), les variétés testées (CK 73, CK90) pourraient être diffusées auprès des paysans en tenant compte des aspects organoleptiques. II est également important de noter que la grande taille des variétés CK73 et CK 90 est un atout pour la gestion des mauvaises herbes et faciliterait la récolte manuelle au niveau des paysans.

pour le financement des travaux ainsi que les riziculteurs du site de Petit Bouaké à Yamoussoukro. 


\section{REFERENCES}

Abdoul K. C., 2006. Testing and developing tolerant rice varieties to iron toxicity in lower guinea (CRA Kilissi and Koba). In Audebert A., Narteh L.T., Kiepe P., Millar D., Beks B. Eds., Iron Toxicity in Rice-based system in West Africa. WARDA, Cotonou, 64-74.

Amancho A. N., Kouassi N. K., Atta H. D., Bouet A., Aidara D. et Sangaré A., 2008. Epidémiologie de la panachure jaune du riz : distribution et incidence sur les variétés de riz (oryza sativa) cultivées en Côte d'Ivoire. Agronomie Africaine 20 (2) : $201-211$.

Amancho A.N., Diallo A.H., Kouassi K.N., Bouet A. et N'guessan P., 2009. Criblage de quelques variétés de riz de Côte d'lvoire pour la résistance à la panachure jaune du riz : Incidence de la maladie sur quelques caractères agronomiques. Sciences et Nature, 6(1): 27-37.

ANADER : 2004. Bilan annuel de la zone de l'Agence Nationale de Développement Rural

(ANADER), zone de Yamoussoukro, Côte d'Ivoire.

Asch F., Becker M. and Kpongor D. S., 2005. A quick and efficient screen for resistance to iron toxicity in lowland rice. J. Plant Nutr. Soil Sci. 168 : 764773.

Audebert A. 2006a. Diagnostic du risque et approche de gestion de la toxicité ferreuse dans les basfonds rizicoles. In Toxicité Ferreuse dans les Systèmes à base riz d'Afrique de l'Ouest, Audebert A, Narteh LT, Kiepe P, Millar D, Beks $B$ (Eds.). Centre du riz pour l'Afrique (ADRAO) Cotonou, Bénin. Cirad-CA UPR Rizicultures : France Montpellier ; 6 -17.

Audebert A. 2006b. La toxicité ferreuse. Les conditions environnementales et les symptômes. In Toxicité Ferreuse dans les Systèmes à Base Riz d'Afrique de l'Ouest, Audebert A, Narteh LT, Kiepe $P$, Millar D, Beks $B$ (Eds). Centre du riz pour l'Afrique (ADRAO) Cotonou, Bénin. CiradCA UPR Rizicultures : France Montpellier ; 1834.

Becker M. and Asch F., 2005. Iron toxicity in rice condition and management concepts. J. Plant Nutri. Soil Sci., 168: 558-573.

Bouet A. 2008. Contribution à la lutte contre la pyriculariose, maladie fongique du riz (Oryza sativa L.) en Côte d'Ivoire : caractéristiques de la population de l'agent pathogène (Magnaporthe grisae) et méthodes d'évaluation variétale pour la résistance à la pyriculariose foliaire et à la pyriculariose du cou. Thèse présentée à l'UFR Biosciences en vue de l'obtention du Doctorat. Université de Cocody, Côte d'Ivoire. 114p.

Bouet A., Gbedie N. A., Boka A. et Kouassi N., 2015. Évaluation des variétés de riz prometteuses pour la résistance à quelques contraintes biotiques majeures et pour leurs performances agronomiques en Côte d'lvoire. Int. J. Biol. Chem. Sci. 9(4): 2041-2056.

Chérif M., Zouzou M., Fofana M. et Audebert A., 2007. Effet de la toxicité du fer sur l'activité photosynthétique du riz. Agronomie Africaine 19 (2): 161-172.

Chérif M., Audebert A., Fofana M. and Zouzou M., 2009. Évaluation of iron toxicity on lowland irrigated rice in West Africa. Tropicultura 27 (2) : 88-92.

Ethan S., Odunze A. C., Abu S. T. and Iwuafor E. N. O., 2011. Effect of water management and nitrogen rates on iron concentration and yield in lowland rice. Agric. Biol. J. N. Am. 2(4): 622-629.

Fageria N. K., Santos N. A. B., Barbosa M. P. et Quimanges C. M., 2008. Iron toxicity in lowland rice. J. Plant Nutr., 31(9):1676-1699.

FAOstat : 2014. Production-Crops, Food and Agriculture Organization of the United Nation. www.faostat.fao.org

Hodomihou R. N., Agbossou E. K., Amadji G. L. et Nacrao H. B., 2011. Effets de différentes doses de phosphate naturel sur la réduction de la toxicité

ferreuse des sols du bas-fond de Niaouli au sud Benin. Int. J. Biol. Chem. Sci. 5(6): 2278-2290.

IRRI: 2013. Standard evaluation system for rice. 5th ed. International Rice Research Institute, Manila, The Philippines.

Odjo T., Kawasaki-Tanaka A., Noda T., Ahohuendo B. C., Séré Y., Kumashiro T., Yanagihara S. and Fukuta S., 2014. Pathogenicity Analysis of Blast (Pyricularia oryzae Cavara) Isolates from West Africa. JARQ 48 (4): 403-412.

Onaga G., Edema R. and Asea G., 2013. Tolerance of rice germplasm to iron toxicity stress and the relationship between tolerance, $\mathrm{Fe}^{2+}, \mathrm{P}$ and $\mathrm{K}$ 
content in the leaves and roots. Arch. Agron. Soil Sci. 59 :213-229.

ONDR: 2016. Office National de développement de la riziculture. www.ondr.ci

Ouedraogo S. et Ouedraogo M., 2003. Évaluation économique de l'impact de la recherche et de la vulgarisation sur le riz au Burkina-Faso. Minireport, Institut de l'environnement et des recherches agricoles (INERA), Ouagadougou, 124.

Séré Y., Fargette D., Abo M. E., Wydra K., Bimerew M., Onasanya A. and Akator S. K., 2013. Managing the Major Diseases of Rice in Africa. In Realizing Africa's rice promise. Wopereis M.C.S et al. (Eds.). CAB International, 213-228.

Sikirou M., Saito K., Achigan-Dako E. G., Dramé K. N., Adam A. and Venuprasad R., 2015. Genetic improvement of iron toxicity tolerance in riceprogress, challenges and prospects in West Africa. Plant Production Science 18(4): 423-434.

WARDA: 2002. Toxicité ferreuse dans les bas-fonds: la rouille du riz. West Africa Rice Development Association, Bouaké, rapport annuel 2001-2002, 29-37.

WARDA: 2006. Iron toxicity in rice-based system in West Africa. West Africa Rice Development Association, Cotonou.1-175. 